\title{
Evaluation of a screener to assess diet quality in the Netherlands
}

\author{
Linde van Lee, Edith J. M. Feskens, Saskia Meijboom, Eveline J. C. Hooft van Huysduynen, \\ Pieter van't Veer, Jeanne H. M. de Vries and Anouk Geelen* \\ Division of Human Nutrition, Wageningen University, P.O. Box 8129, 6700 EV Wageningen, The Netherlands
}

(Submitted 14 July 2015 - Final revision received 18 October 2015 - Accepted 27 October 2015 - First published online 2 December 2015)

\section{Abstract}

Generally, there is a need for short questionnaires to estimate diet quality in the Netherlands. We developed a thirty-four-item FFQ - the Dutch Healthy Diet FFQ (DHD-FFQ) - to estimate adherence to the most recent Dutch guidelines for a healthy diet of 2006 using the DHD-index. The objectives of the present study were to evaluate the DHD-index derived from the DHD-FFQ by comparing it with the index based on a reference method and to examine associations with participant characteristics, nutrient intakes and levels of cardiometabolic risk factors. Data of 1235 Dutch men and women, aged between 20 and 70 years, participating in the Nutrition Questionnaires plus study were used. The DHD-index was calculated from the DHD-FFQ and from a reference method consisting of a 180-item FFQ combined with a 24-h urinary Na excretion value. Ranking was studied using Spearman's correlations, and absolute agreement was studied using a Bland-Altman plot. Nutrient intakes derived from the 180-item FFQ were studied according to quintiles of the DHD-index using DHD-FFQ data. The correlation between the DHD-index derived from the DHD-FFQ and the reference method was 0.56 (95\% CI 0.52, 0.60). The Bland-Altman plot showed a small mean overestimation of the DHD-index derived from the DHD-FFQ compared with the reference method. The DHD-index score was in the favourable direction associated with most macronutrient and micronutrient intakes when adjusted for energy intake. No associations between the DHD-index score and cardiometabolic risk factors were observed. In conclusion, the DHD-index derived from the DHD-FFQ was considered acceptable in ranking but relatively poor in individual assessment of diet quality.

\section{Key words: Validity studies: Dietary patterns: Dietary guidelines: FFQ}

Nutrition is an important risk factor in the development of chronic diseases such as CVD, diabetes and several cancers ${ }^{(1)}$. To decrease the risk of chronic diseases, dietary guidelines were developed based on scientific evidence ${ }^{(2)}$. When developing public health interventions or health education programmes, it is important to monitor the adherence to dietary guidelines. Moreover, monitoring adherence to dietary guidelines can also be useful for identification of individuals with a low diet quality in clinical settings such as the general practitioner's practice.

Recently, the Dutch Healthy Diet index (DHD-index) was developed that comprises ten components representing the current ten quantitative Dutch guidelines for a healthy diet of 2006 on physical activity, vegetables, fruit, dietary fibre, fish, consumption occasions with acidic drinks and foods, SFA, trans-fatty acids (TFA), Na and alcohol ${ }^{(2,3)}$. As the DHD-index score was in the favourable direction associated with several micronutrient intakes, and biomarkers of dietary intake ${ }^{(3,4)}$, it was considered a useful tool to assess diet quality in the Dutch population. Calculation of the DHD-index requires data on dietary intake, for instance, assessed by multiple 24-h recalls, food diaries or a FFQ. Unfortunately, these dietary assessment methods are time consuming, and therefore less likely to be used in everyday clinical and public health practice. In these situations, there is a need for a method that quickly assesses the diet quality of individuals ${ }^{(5)}$. To date, short food questionnaires have been developed for the Mediterranean $\operatorname{diet}^{(6,7)}$, for the American $\operatorname{diet}^{(8)}$ and the Dietary Approaches to Stop Hypertension diet ${ }^{(9)}$. However, no fast method to assess diet quality according to the Dutch dietary guidelines was available. Therefore, we developed a screener, entitled the DHD-FFQ, to estimate the DHD-index score for ranking individuals based on their diet quality.

The objective of the present study was to evaluate the DHD-index derived from the DHD-FFQ. For this, we examined correlations and the absolute agreement between the DHD-index and its components based on the DHD-FFQ compared with those based on a reference method. The reference method consisted of a full-length FFQ combined with a 24-h urinary $\mathrm{Na}$ value. Correlation coefficients of 0.4 or higher were previously considered acceptable ${ }^{(6,8,10)}$, in studies comparing screeners with a 137 -item FFQ or multiple 24-h recalls. Second, we examined associations of the DHD-index based on the

Abbreviations: DHD, Dutch Healthy Diet; DNFCS, Dutch National Food Consumption Survey; PABA, para-aminobenzoic acid; TFA, trans-fatty acids.

* Corresponding author: A. Geelen, email Anouk.Geelen@wur.nl 
DHD-FFQ with participants' characteristics and energy and nutrient intakes derived from the full-length FFQ. Finally, we compared the associations between cardiometabolic risk factors and the DHD-index score derived from the DHD-FFQ with the score derived from the reference method.

\section{Methods}

\section{Study population and design}

The Nutrition Questionnaires plus (NQplus) study is a 3-year observational study in the general Dutch population. It was designed for multiple aims: to validate a newly developed FFQ, to start a reference database for nutrition research and to study associations between diet and intermediate health outcomes. Between May 2011 and December 2013, recruitment was carried out by sending letters and emails to randomly selected inhabitants of Wageningen, Renkum, Ede, Arnhem and to all households in Veenendaal. Inclusion criteria for the study were as follows: aged between 20 and 70 years and able to speak and write Dutch. This study was conducted according to the guidelines laid down in the Declaration of Helsinki, and all procedures involving the participants were approved by the Medical Ethics Committee of Wageningen University. Written informed consent was obtained from all participants.

Baseline measurements consisted of dietary assessment (FFQ and 24-h recalls), physical measurements (e.g. height, weight, blood pressure), venepuncture, a 24-h urinalysis, general questionnaires (e.g. demographics, history of disease) and lifestyle questionnaires including questions about physical activity (Squash) ${ }^{(14)}$. Physical activity level was classified as adherent or non-adherent to the Dutch physical activity guideline of being moderately physically active for at least $5 \mathrm{~d} /$ week for $30 \mathrm{~min}^{(2)}$. Medication use was determined and classified according to the Anatomical Therapeutic Chemical (ATC) classification system.

For the present study, dietary data from the FFQ were used. Participants self-administered the DHD-FFQ and the full-length FFQ using the open source survey tool LimeSurvey ${ }^{\mathrm{TM}}$ (LimeSurvey Project Team/Carsten Schmitz, 2012). The 180-item FFQ was either administered 1 month after the start of the study (63\%) or in July-August 2013 (37\%). The DHD-FFQ was administered between June and October 2013. The FFQ were administered with at least 1 month in between (median range: 3.4 months, interquartile range: 11.9 months). Order of administration was dependent on progress within the NQplus study, resulting in $27 \%$ of the participants filling out the DHD-FFQ before the 180-item FFQ.

\section{Dietary assessment}

Dutch Healthy Diet FFQ. The DHD-FFQ was developed using the Dutch FFQ-TOOL ${ }^{\mathrm{TM}(15)}$ using data of the Dutch National Food Consumption Survey (DNFCS) 2007-2010 ${ }^{(16)}$ as reference. This tool was developed to generate and process FFQ using reproducible and standardised procedures based on the percentage absolute contribution and explained variance in nutrient intake ${ }^{(15)}$. The DHD-index components 'physical activity' and 'consumption occasions with acidic drinks and foods' cannot be assessed with most FFQ; therefore, we did not take these components into account during development of the DHD-FFQ. The food items that contributed most to the level of the following nutrient intakes were selected: dietary fibre, SFA, TFA and Na. This procedure of selecting food items based on their percentage contribution to the absolute nutrient intake in a reference population was suggested by Block et $a l^{(17,18)}$ and refers to the first MOMent (MOM1) of the intake of a nutrient ${ }^{(19)}$. In addition, the food items representing the food groups vegetables, fruit, fish and alcohol were included. Furthermore, $\mathrm{Na}$ intake was separated into two parts: $\mathrm{Na}$ intake from foods and discretionary salt; thus, two questions on discretionary $\mathrm{Na}$ were included to estimate the frequency of salt or Na-rich products (i.e. soya sauce and soup flavouring) added during cooking and at the table. This resulted in a list of thirty-four food items, which together accounted for $73 \%$ of total dietary fibre intake, $70 \%$ of total SFA intake, $81 \%$ of total TFA intake and $73 \%$ of total $\mathrm{Na}$ from food intake within the adult population of the DNFCS 2007-2010 (online Supplementary Table S1). The percentage between-person variability explained by the selected thirty-four food items, the so-called second MOMent (MOM2) of the nutrient intake distribution ${ }^{(19)}$, was $55 \%$ for dietary fibre, $58 \%$ for SFA, $47 \%$ for TFA and $65 \%$ for Na. In total, the DHD-FFQ comprises twenty-five questions, representing the thirty-four items, on intakes of bread, fruit, vegetables, potatoes, milk, cheese, meat, meat products, fish, cookies, pastries, crisps, soup, fats and oils, Asian foods, pizza, alcohol and added $\mathrm{Na}$. The answer categories for the frequency questions ranged from 'never' to 'every day' offering six options. The reference period was the previous month. Portion sizes were assessed in standard portions, natural portions or commonly used household measures such as glasses or bowls. Nutrient content per food item was estimated by calculating a weighted average of the individual nutrient content of foods and drinks included in the food item using the 2011 Dutch Food Composition Table ${ }^{(20)}$ and the consumption data from the DNFCS 2007-2010. These nutrient contents were subsequently multiplied with the portion sizes and the frequency of intake to estimate nutrient intakes. All food items were used to calculate the intakes of dietary fibre, SFA, TFA and Na. The face validity of questions and answer categories was evaluated in a research panel of 688 Dutch persons. On the basis of these results, questions were optimised. Mean time to complete the DHDFFQ, estimated by the online survey tool, was 7.8 (SD 5.6) min in these 688 persons and was considered acceptable.

Full-length FFQ. The 180-item semi-quantitative FFQ was used to assess habitual dietary intake, and the reference period was the previous month. This FFQ was validated against actual energy intake to maintain body weight for energy intake $(r 0.82)^{(11)}$, and validated against three 24-h recalls for SFA $(r 0 \cdot 44)$, TFA $(r 0 \cdot 42)$, vegetables $(r 0 \cdot 24)$, fruit $(r 0 \cdot 50)$, fish ( $r$ 0.37), alcoholic beverages $\left(\begin{array}{ll}r & 0.78)\end{array}\right)$ and dietary fibre $(r 0.66)^{(13)}$. Answer categories for frequency questions ranged from 'never per month' to ' $6-7 \mathrm{~d} /$ week' offering seven options, and portion sizes were estimated using standard portions, natural 
portions and commonly used household measures. Nutrient content per food item was estimated by calculating a weighted average of the individual nutrient content of foods and drinks included in the food item using the 2011 Dutch Food Composition Table ${ }^{(16)}$ and consumption data from the DNFCS $1998^{(21)}$. These nutrient contents were multiplied with the portion sizes and the frequency of intake to estimate nutrient intakes.

24-h urinary sodium. Na intake was assessed using 24-h urine samples - the gold standard method ${ }^{(22)}$. Para-aminobenzoic acid (PABA) was used to check for completeness of the urinary collections and measured using HPLC method ${ }^{(23)}$ (laboratory Division of Human Nutrition, Wageningen University, The Netherlands). PABA is assumed to be excreted almost quantitatively within $24 \mathrm{~h}$, and a urine sample with a recovery of at least $78 \%(187 \mathrm{mg})$ of the $3 \times 80 \mathrm{mg}$ ingested PABA was considered a complete urine collection ${ }^{(23)}$. Nine urine samples with PABA recoveries $<50 \%$ were excluded from the data analyses. Recoveries between 50 and $78 \%$ ( $n$ 121) were proportionally adjusted to the mean PABA recovery of $88 \%$ using linear regression equations as suggested by others ${ }^{(24,25)}$.

Urinary $\mathrm{Na}$ level was measured using an ion-selective electrode from a Roche/Hitachi 917 analyser (Roche) ${ }^{(22)}$ at SHO in Velp, the Netherlands. Total 24-h Na excretion was calculated by multiplying total weight of collected urine by $\mathrm{Na}$ concentration. In addition, this was divided by $0 \cdot 86$, assuming that $86 \%$ of $\mathrm{Na}$ intake is excreted via urine ${ }^{(26)}$

\section{Dutch Healthy Diet index}

The scoring for the DHD-index has been described in detail elsewhere $^{(3,4)}$ and has been summarised in Table 1. In brief, for the components vegetables, fruit, dietary fibre and fish, no intake resulted in a component score of 0 points. Intakes equal to or above the cut-off values representing the dietary guidelines received the maximum of 10 points. For the components SFA, TFA, Na and alcohol, intakes below the cut-off values received the maximum of 10 points. A score of 0 points was assigned when intake was higher than the binge drinking threshold values for the alcohol component ${ }^{(27)}$ or higher than the threshold values representing the 85th percentiles of the intakes of a Dutch reference population ${ }^{(16)}$ for the components SFA, TFA and $\mathrm{Na}$. Scores between 0 and 10 points were calculated proportionally, except for TFA that was scored dichotomously. The summed total score could range from 0 (no adherence) to 80 (complete adherence).

The Na component score derived from the full-length FFQ was based on a single 24-h $\mathrm{Na}$ excretion. Na excretions $\leq 2400 \mathrm{mg}$ were assigned 10 points, and excretions exceeding $3600 \mathrm{mg}$ were assigned 0 points.

As the DHD-FFQ was not designed to estimate total energy intake, the DHD-index scoring had to be slightly adapted for the components expressed in energy percentages when it was applied to the DHD-FFQ data. The cut-off and threshold values for the energy-dependent components dietary fibre, SFA and TFA were calculated using sex-specific average energy requirements $(10.5 \mathrm{MJ} \text { for men and } 8.4 \mathrm{MJ} \text { for women })^{(28)}$. These sex-specific average energy requirements and $\mathrm{Na}$ cut-off values were proportionally lowered, matching the percentage coverage of total energy intake as assessed by the DHD-FFQ (6.7 MJ for men and $5.4 \mathrm{MJ}$ for women), to arrive at cut-off and threshold values that were appropriate for the estimated dietary intake assessed by the DHD-FFQ (Table 2). Furthermore, scores for $\mathrm{Na}$ intake were separated into two parts. The answers on discretionary $\mathrm{Na}$ contributed 3 points based on the assumption that about $30 \%$ of total $\mathrm{Na}$ intake is from added salt ${ }^{(29,30)}$. Na intake from foods contributed to the remaining 7 points. Finally, the fish component score was based on the frequency of lean or fatty fish intake instead of fish fatty acid intake.

\section{Cardiometabolic risk factor assessment}

Physical examination. All physical measurements were performed by trained research assistants following a standardised protocol. Height and waist circumference were measured to the nearest $0.5 \mathrm{~cm}$ using a stadiometer (SECA 213; SECA Corp.)

Table 1. Cut-off and threshold values for calculation of the Dutch Healthy Diet index component scores for the reference method and the DHD-FFQ

\begin{tabular}{|c|c|c|c|c|}
\hline & \multicolumn{2}{|c|}{ Reference method ${ }^{*}$} & \multicolumn{2}{|c|}{ DHD-FFQ } \\
\hline & Minimum points $(0)$ & Maximum points (10) & Minimum points $(0)$ & Maximum points (10) \\
\hline Vegetables & $0 \mathrm{~g}$ & $\geq 200 \mathrm{~g}$ & $0 \mathrm{~g}$ & $\geq 200 \mathrm{~g}$ \\
\hline Fruit & $0 \mathrm{~g}$ & $\geq 200 \mathrm{~g}$ & $0 \mathrm{~g}$ & $\geq 200 \mathrm{~g}$ \\
\hline Fibre & $0 \mathrm{~g} / 4 \cdot 2 \mathrm{MJ}$ & $\geq 14 \mathrm{~g} / 4.2 \mathrm{MJ}$ & $0 \mathrm{~g}$ & $\begin{array}{l}\delta: \geq 22.40 \mathrm{~g} \dagger \\
\text { o: } \geq 17.92 \mathrm{~g} \dagger\end{array}$ \\
\hline Fish & $0 \mathrm{mg} E P A+D H A$ & $\geq 450 \mathrm{mg}$ EPA + DHA & 0 times fish/week & 2 times fish/week \\
\hline SFA & $\geq 15$ en\% & $<10$ en\% & $\begin{array}{l}\text { o: } \geq 26.67 \mathrm{~g} \\
\text { \&: } \geq 21.33 \mathrm{~g}\end{array}$ & $\begin{array}{l}\text { o: }<17.78 \mathrm{g \dagger} \\
\text { ᄋ: }<14.22 \mathrm{g \dagger}\end{array}$ \\
\hline TFA & $\geq 1$ en $\%$ & $<1$ en $\%$ & $\begin{array}{l}\text { o: } \geq 1.78 \mathrm{~g} \\
\text { \%: } \geq 1.42 \mathrm{~g}\end{array}$ & $\begin{array}{l}\text { o: }<1.78 \mathrm{g \dagger} \\
\text { o: }<1.42 \mathrm{g \dagger}\end{array}$ \\
\hline $\mathrm{Na}$ & $\geq 3600 \mathrm{mg}$ & $<2400 \mathrm{mg}$ & $\geq 2304 \mathrm{mg} \ddagger$ & $<1536$ mg†‡ \\
\hline Alcohol & o: $\geq 60 \mathrm{~g}$ ethanol & o: $\leq 20 \mathrm{~g}$ ethanol & o: $\geq 6$ drinks & $0: \leq 2$ drinks \\
\hline & o: $\geq 40 \mathrm{~g}$ ethanol & $: \leq 10 \mathrm{~g}$ ethanol & $: \geq 4$ drinks & q: $\leq 1$ drink \\
\hline
\end{tabular}

TFA, trans-fatty acids.

* Full-length FFQ combined with a 24-h urinary Na value.

$\dagger$ Cut-off values were calculated with sex-specific average energy requirements $\times$ coverage of energy intake assessed by the DHD-FFQ ( 0 : $6.7 \mathrm{MJ}$, o: $5.4 \mathrm{MJ}$ ).

$\ddagger \mathrm{Na}$ from foods accounted for a maximum of 7 points, and discretionary $\mathrm{Na}$ for a maximum of 3 points. 
Table 2. Dutch Healthy Diet index (DHD-index) and its component scores using reference data and DHD-FFQ data in 1235 participants of the Nutrition Questionnaires plus study

(Mean values and standard deviations; Kendall's tau-b coefficients, Spearman's correlations and $95 \%$ confidence intervals)

\begin{tabular}{|c|c|c|c|c|c|c|c|c|}
\hline & \multicolumn{2}{|c|}{ Reference method ${ }^{*}$} & \multicolumn{2}{|c|}{ DHD-FFQ } & \multirow[b]{2}{*}{ Tau-b } & \multirow[b]{2}{*}{$95 \% \mathrm{Cl}$} & \multirow[b]{2}{*}{$R$} & \multirow[b]{2}{*}{$95 \% \mathrm{Cl}$} \\
\hline & Mean & SD & Mean & SD & & & & \\
\hline DHD-index† & 54.0 & $10 \cdot 1$ & $57 \cdot 6$ & $9 \cdot 6$ & 0.40 & $0.37,0.43$ & 0.57 & $0.53,0.60$ \\
\hline Vegetables & $7 \cdot 0$ & $2 \cdot 7$ & $6 \cdot 7$ & $2 \cdot 6$ & 0.39 & $0.36,0.42$ & 0.54 & $0.50,0.58$ \\
\hline Fruit & $7 \cdot 2$ & 3.4 & $8 \cdot 0$ & $2 \cdot 7$ & 0.54 & $0.51,0.58$ & 0.66 & $0.63,0.69$ \\
\hline Fibre & $8 \cdot 2$ & 1.4 & $7 \cdot 8$ & 1.9 & 0.22 & $0.18,0.26$ & 0.31 & $0.26,0.36$ \\
\hline Fish & $4 \cdot 1$ & 2.9 & 5.5 & $3 \cdot 2$ & 0.46 & $0.43,0.49$ & 0.61 & $0.57,0.64$ \\
\hline SFA & $6 \cdot 1$ & $3 \cdot 4$ & $5 \cdot 5$ & $4 \cdot 0$ & 0.28 & $0.24,0.32$ & 0.38 & $0.33,0.43$ \\
\hline TFA & $9 \cdot 9$ & $1 \cdot 0$ & $9 \cdot 2$ & $2 \cdot 7$ & 0.09 & $-0.01,0.19$ & 0.09 & $0.03,0.14$ \\
\hline $\mathrm{Na}$ & $2 \cdot 4$ & $3 \cdot 7$ & $6 \cdot 3$ & $2 \cdot 8$ & $0 \cdot 18$ & $0.14,0.23$ & 0.24 & $0.19,0.29$ \\
\hline Alcohol & $9 \cdot 2$ & 1.9 & $8 \cdot 6$ & $2 \cdot 7$ & 0.53 & $0.48,0.57$ & 0.58 & $0.54,0.61$ \\
\hline
\end{tabular}

TFA, trans-fatty acids.

${ }^{*}$ Full-length FFQ combined with a urinary Na value.

$\dagger$ Excluding the components physical activity and consumption occasions with acidic drinks and foods.

and a non-flexible tape, respectively. Participants' weight was measured to the nearest $0 \cdot 1 \mathrm{~kg}$ on a digital scale while wearing light clothing without shoes (SECA 877 (SECA Corp.) or BC418MA (Tanita Corp.). After $10 \mathrm{~min}$ of rest, blood pressure (IntelliSense HEM-907; Omron Healthcare) was measured six times on the left arm with 2 min of rest in between the measurements. The first blood pressure measurement was discarded for validity reasons, and the remaining five blood pressure measurements were averaged.

Blood sampling and analyses. Participants underwent a venepuncture in the fasting state at the hospital in Ede or Velp, The Netherlands. Blood sample analyses were carried out in the accompanying hospital laboratories. Both laboratories joined an external quality control programme and used the same methodology and protocols for risk factor assessments. Total cholesterol, HDL-cholesterol and fasting TAG levels were determined with enzymatic methods $^{(31)}$ using a Siemens Dimension Vista 1500 automated analyser (Siemens) in Ede and a Hitachi Modular P800 Chemistry Analyser (Hitachi Group) in Velp. HbA1C was determined with HPLC measurement technology using an ADAMS A1c 8160 analyser (Siemens) at both locations.

\section{Data analyses}

Complete data from the 180-item FFQ, the DHD-FFQ and Na excretion were available for 1247 participants (Fig. 1). We excluded twelve participants who were pregnant or had a non-fasting blood sample, resulting in a total sample of 1235 participants.

Mean and standard deviation scores of the DHD-index and its components calculated from the DHD-FFQ and the reference method are presented. Kendall's tau-b coefficients were calculated between scores derived from the reference method and from the DHD-FFQ to examine ranking of participants. Spearman's correlations are shown to allow comparison with previous studies. CI for these correlations were obtained using Fisher's $z$-transformation. Agreement of the DHD-index score between the two methods was examined using a Bland-Altman plot ${ }^{(32)}$.
Participants' characteristics and energy, macronutrient and micronutrient intakes estimated from the full-length FFQ were examined according to quintiles of the DHD-index score derived from the DHD-FFQ. We adjusted macronutrient and micronutrient intakes for energy intake estimated from the full-length FFQ as well as for energy intake estimated from the DHD-FFQ. The rationale for adjustment for energy estimated from the DHD-FFQ was to study the ability of using energy intake as covariate for future use. Linear trends across the quintiles were examined using general linear models.

Furthermore, partial Spearman's correlations between the DHD-index and the cardiometabolic risk factors total cholesterol, HDL-cholesterol, TAG, HbA1C and systolic and diastolic blood pressures were examined adjusted for age, sex, smoking (never, former, current) and adherence to physical activity guidelines (yes/no). We additionally adjusted for BMI as a potential intermediate. Missing values for the covariates education level ( $n$ 11), smoking ( $n$ 86) and physical activity ( $n$ 90) were imputed for five times, and results were pooled using the MIANALYZE procedure in SAS. Participants using medication for diabetes mellitus, hypertension or dyslipidaemia (ATC codes: A10, C1-C9, C10) were excluded from the analyses when examining the association between diet quality and cardiometabolic risk factors ( $n 29,180,110$, respectively). All the analyses were carried out using SAS statistical software version 9.3 (SAS Institute Inc.), and statistical significance was set at $P<0.05$.

\section{Results}

The mean DHD-index score based on DHD-FFQ data was 57.6 (SD 9.6) out of a possible total score of 80 points, and was similar to the score using the reference method consisting of a full-length FFQ combined with a urinary Na value (mean 54.0 (SD 10.1); Table 2). The Kendall's tau-b coefficients between the two scores was 0.40 (95\% CI $0.37,0.43)$. This correlation was similar when adjusting for order of occurrence or time between the 180-item FFQ and the DHD-FFQ (data not shown). When comparing the mean DHD-index component scores between the reference method and the DHD-FFQ, the smallest absolute difference was seen for the fibre component ( $8.2 v .7 \cdot 8$ points) and the largest 


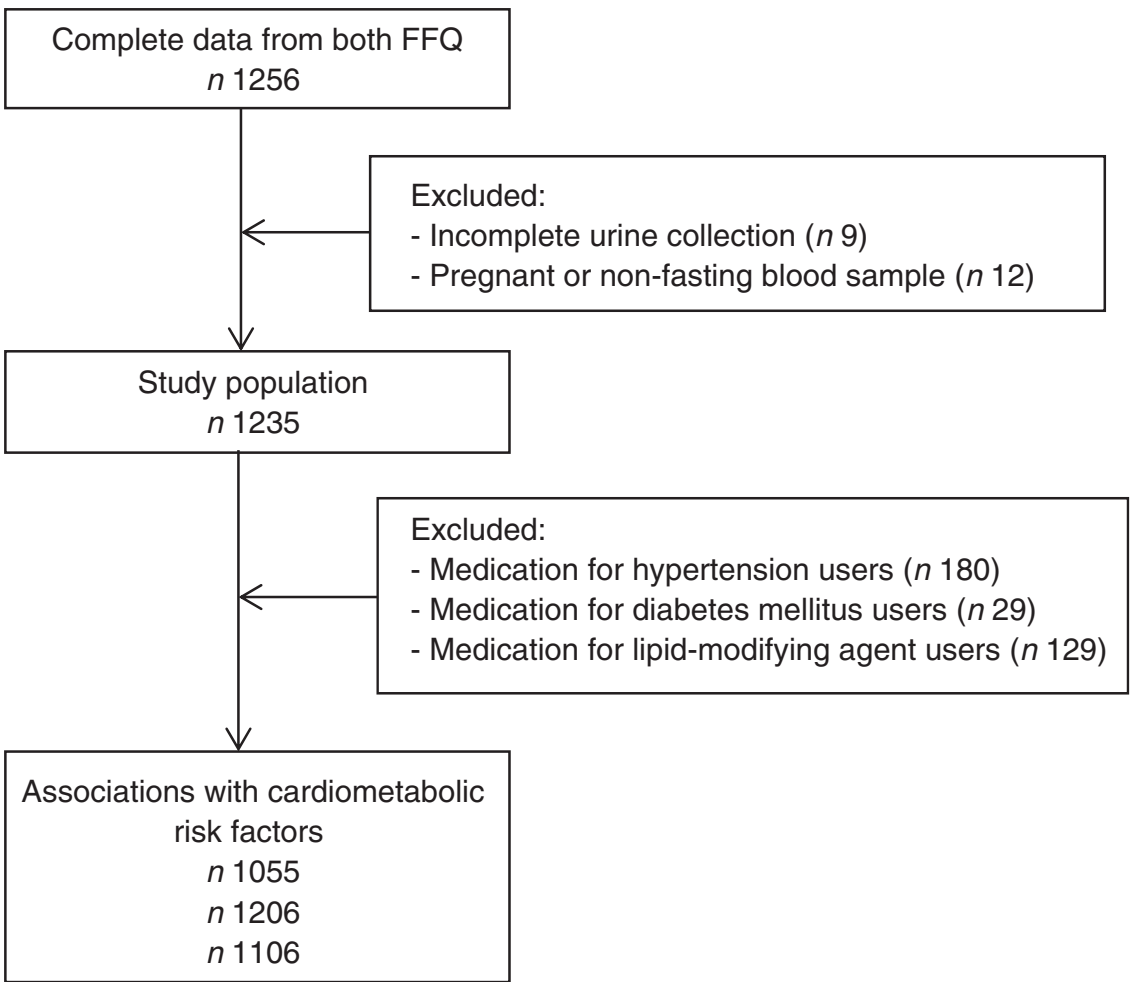

Fig. 1. Flow diagram on exclusion of participants of the Nutrition Questionnaires plus study.

absolute difference was seen for the $\mathrm{Na}$ component $(2 \cdot 4 \mathrm{v}$. 6.3 points). The lowest tau-b coefficient was observed for the component TFA (0.09; $95 \%$ CI -0.01, 0.19). For the components fibre, SFA and $\mathrm{Na}$, tau-b correlations ranged between $0 \cdot 2$ and $0 \cdot 4$, whereas for the components vegetables, fruit, fish and alcohol the correlations were 0.4 and higher.

Absolute agreement was studied using a Bland-Altman plot and accompanying limits of agreement (Fig. 2). The mean difference, the DHD-index based on the reference method minus the DHD-index based on the DHD-FFQ, was -3.6 points, and limits of agreement were -21.7 and 14.5 points. The DHD-index based on the DHD-FFQ showed an overestimation in the lower scores and an underestimation in the higher scores when compared with the DHD-index based on the reference method.

Positive associations were observed between the DHD-index derived from DHD-FFQ data and age $\left(P_{\text {for }}\right.$ trend $\left.<0.001\right)$, following a diet regimen $\left(P_{\text {for }}\right.$ trend $\left.=0.001\right)$, supplement use $\left(P_{\text {for }}\right.$ trend $\left.<0.01\right)$ and antihypertensive medication use ( $P_{\text {for trend }}=0.035$; Table 3 ). Furthermore, participants in the higher quintiles were less likely to be men than those in the lower quintiles. For the participant characteristics BMI, smoking, education level, adherence to the physical activity guideline and use of lipid-modifying drugs and diabetic drugs, no association with the DHD-index was observed.

Intakes of energy, carbohydrates, protein, total fat and alcohol derived from the full-length FFQ were inversely associated with the DHD-index score based on DHD-FFQ, whereas dietary fibre intake was positively associated with the DHD-index score (Table 4). When additionally adjusted for energy intake estimated from the full-length FFQ, the intake of total fat and alcohol remained inversely associated with the DHD-index, whereas the intake of carbohydrates and fibre became positively associated. Similar trends were observed when adjusted for energy intake estimated from the DHD-FFQ, except for carbohydrates, where the positive association disappeared.

Regarding the intakes of micronutrients, $\mathrm{Ca}$, vitamin $\mathrm{A}$, vitamin $B_{1}$, vitamin $B_{2}$ and vitamin $B_{12}$ were inversely associated with the DHD-index score, whereas the micronutrients folate and vitamin $\mathrm{C}$ were positively associated and vitamin $\mathrm{E}$ was not associated (Table 5). When additionally adjusted for energy intake estimated from the full-length FFQ, the association with intakes of $\mathrm{Ca}$ and vitamin A disappeared, whereas all other micronutrients became positively associated across quintiles of the DHD-index score. Similar positive trends were observed for most micronutrients, when adjusted for energy intake estimated from the DHD-FFQ.

The DHD-index score derived from the reference method was inversely associated with fasting TAG $(P<0.01$; online Supplementary Table S2) and HbA1C $(P<0.05)$ when adjusted for age, sex, smoking, physical activity level and energy intake. After additional adjustment for BMI, the associations disappeared. The DHD-index derived from the DHD-FFQ was borderline inversely associated with fasting TAG and HbA1C when adjusted for age, sex, smoking and physical activity $(P<0 \cdot 10)$. After additional adjustment for BMI, the association with fasting TAG disappeared and the association with HbA1C remained similar $(P=0 \cdot 10)$. The results were similar when excluding the participants with missing values for the covariates education, physical activity and smoking (results not shown). 


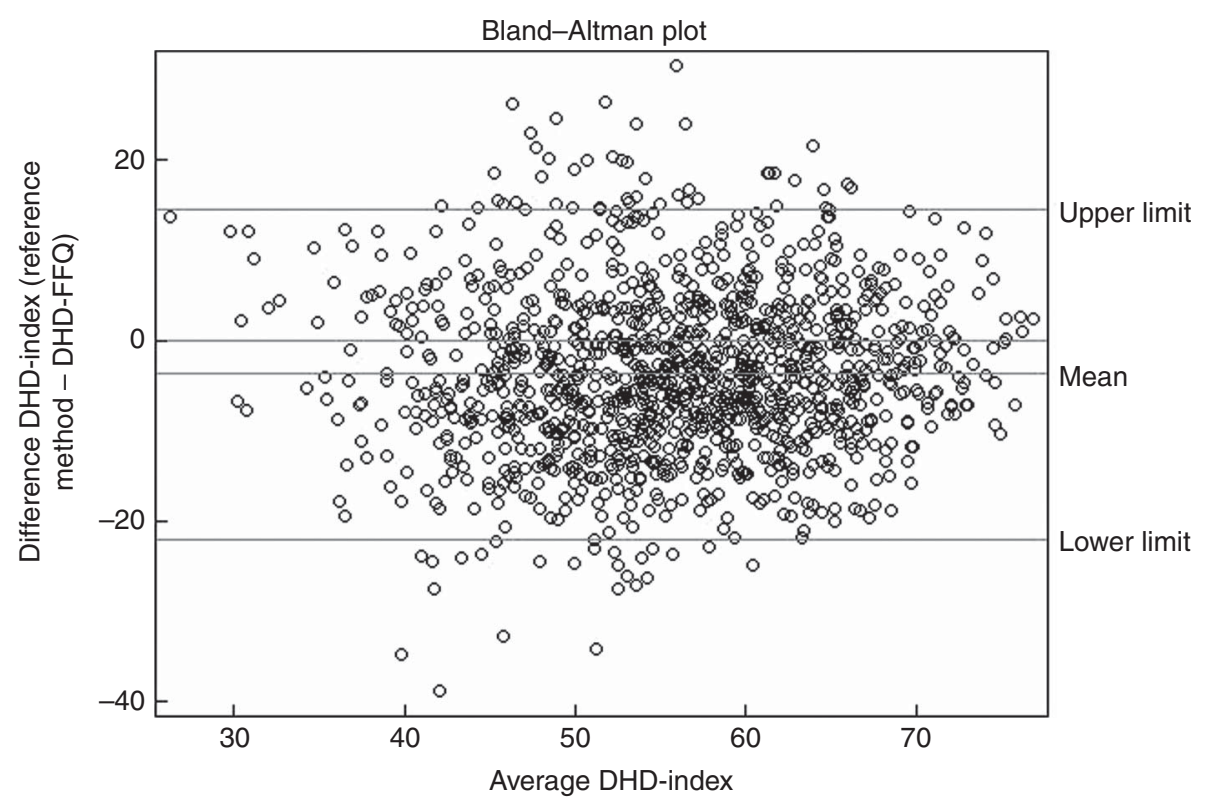

Fig. 2. Bland-Altman plot of the Dutch Healthy Diet index (DHD-index) based on the DHD-FFQ and the DHD-index based on the reference method consisting of a full-length FFQ combined with a urinary Na value in 1235 participants of the Nutrition Questionnaires plus study.

Table 3. Selected characteristics across quintiles (Q) of the Dutch Healthy Diet index (DHD-index) based on the DHD-FFQ in 1235 participants of the Nutrition Questionnaires plus study*

(Numbers and percentages; $n$ 247)

\begin{tabular}{|c|c|c|c|c|c|c|c|}
\hline & \multicolumn{6}{|c|}{ DHD-index derived from the DHD-FFQ } & \multirow[b]{3}{*}{$P_{\text {for trend }}$} \\
\hline & \multicolumn{2}{|c|}{ Q1 } & \multicolumn{2}{|c|}{ Q3 } & \multicolumn{2}{|c|}{ Q5 } & \\
\hline & $n$ & $\%$ & $n$ & $\%$ & $n$ & $\%$ & \\
\hline Men & 139 & $56 \cdot 3$ & 121 & $49 \cdot 0$ & 105 & $42 \cdot 5$ & 0.001 \\
\hline Age (years) & & & & & & & $<0.001$ \\
\hline Mean & \multicolumn{2}{|c|}{50.5} & \multicolumn{2}{|c|}{$54 \cdot 1$} & \multicolumn{2}{|c|}{$55 \cdot 8$} & \\
\hline SD & \multicolumn{2}{|c|}{11.9} & \multicolumn{2}{|c|}{11.3} & \multicolumn{2}{|c|}{$10 \cdot 0$} & \\
\hline BMI $\left(\mathrm{kg} / \mathrm{m}^{2}\right)$ & & & & & & & 0.218 \\
\hline $\begin{array}{l}\text { Mean } \\
\text { SD }\end{array}$ & \multicolumn{2}{|c|}{$\begin{array}{r}25.8 \\
4.1\end{array}$} & \multicolumn{2}{|c|}{$\begin{array}{r}25 \cdot 7 \\
3.6\end{array}$} & \multicolumn{2}{|c|}{$\begin{array}{r}25 \cdot 2 \\
3.8\end{array}$} & \\
\hline Diet regimen & 5 & $2 \cdot 0$ & 8 & $3 \cdot 2$ & 21 & $8 \cdot 5$ & 0.001 \\
\hline Supplement use & 89 & $36 \cdot 0$ & 91 & $36 \cdot 8$ & 125 & $50 \cdot 6$ & $<0.001$ \\
\hline Smoking† & & & & & & & 0.062 \\
\hline Never & 126 & $51 \cdot 0$ & 122 & $49 \cdot 4$ & 121 & $49 \cdot 0$ & \\
\hline Former & 90 & 36.4 & 109 & $44 \cdot 1$ & 112 & $45 \cdot 3$ & \\
\hline Current & 31 & 7.9 & 16 & 6.5 & 14 & $5 \cdot 7$ & \\
\hline Education† & & & & & & & 0.634 \\
\hline Low & 36 & $14 \cdot 6$ & 43 & $17 \cdot 4$ & 36 & $14 \cdot 6$ & \\
\hline Intermediate & 70 & $28 \cdot 3$ & 70 & $28 \cdot 3$ & 73 & $29 \cdot 6$ & \\
\hline High & 140 & $56 \cdot 7$ & 134 & $54 \cdot 3$ & 138 & 55.9 & \\
\hline Physical activity $\dagger$ & 119 & $48 \cdot 2$ & 107 & 43.3 & 106 & $42 \cdot 9$ & 0.675 \\
\hline \multicolumn{8}{|l|}{ Medication use } \\
\hline Lipid-modifying drugs & 20 & $8 \cdot 1$ & 23 & 9.3 & 25 & $10 \cdot 1$ & 0.778 \\
\hline Diabetic drugs & 5 & $2 \cdot 0$ & 8 & $3 \cdot 2$ & 5 & $2 \cdot 0$ & 0.895 \\
\hline Antihypertensive drugs & 24 & $9 \cdot 7$ & 33 & $13 \cdot 4$ & 42 & $17 \cdot 0$ & 0.035 \\
\hline
\end{tabular}

* Cut-off values for quintiles: 49.5, 55.8, 60.6, 65.8.

† Frequencies and percentages are estimated based on five imputations.

\section{Discussion}

In the present study, we evaluated the DHD-index derived from the DHD-FFQ and compared it with the DHD-index derived from a full-length FFQ combined with a 24-h urinary Na value. The DHD-FFQ is a screener and was designed to estimate diet quality in time-limited situations such as clinical or public health practice where full-length FFQ are impractical to use. We showed that the DHD-index derived from the DHD-FFQ was acceptably correlated with the DHD-index derived from the reference method. Absolute agreement, as studied by the Bland-Altman plot, showed a small mean overestimation and 
Table 4. Macronutrient intakes estimated from the full-length FFQ across quintiles $(Q)$ of the Dutch Healthy Diet index (DHD-index) based on the DHD-FFQ in 1235 participants of the Nutrition Questionnaires plus study

(Mean values with their standard errors; $n$ 247)

\begin{tabular}{|c|c|c|c|c|c|c|c|}
\hline & \multicolumn{6}{|c|}{ DHD-index derived from the DHD-FFQ } & \multirow[b]{3}{*}{$P_{\text {for trend }}$} \\
\hline & \multicolumn{2}{|c|}{ Q1 } & \multicolumn{2}{|c|}{ Q3 } & \multicolumn{2}{|c|}{ Q5 } & \\
\hline & Mean & SEM & Mean & SEM & Mean & SEM & \\
\hline Energy (MJ) & $9 \cdot 2$ & 0.2 & $8 \cdot 4$ & $0 \cdot 1$ & $7 \cdot 6$ & $0 \cdot 1$ & $<0.001$ \\
\hline \multicolumn{8}{|c|}{ Carbohydrates (g) } \\
\hline Crude & $229 \cdot 6$ & $5 \cdot 0$ & $217 \cdot 9$ & $4 \cdot 3$ & $199 \cdot 6$ & $3 \cdot 8$ & $<0.001$ \\
\hline Model $1^{\star}$ & $210 \cdot 5$ & $1 \cdot 8$ & $219 \cdot 1$ & $1 \cdot 8$ & $220 \cdot 1$ & $1 \cdot 8$ & $<0.001$ \\
\hline Model $2 \dagger$ & $211 \cdot 8$ & $3 \cdot 7$ & $218 \cdot 1$ & 3.6 & $219 \cdot 6$ & $6 \cdot 7$ & 0.163 \\
\hline \multicolumn{8}{|l|}{ Protein (g) } \\
\hline Crude & $77 \cdot 8$ & 1.4 & 72.5 & $1 \cdot 3$ & $67 \cdot 9$ & $1 \cdot 2$ & $<0.001$ \\
\hline Model $1^{\star}$ & $72 \cdot 5$ & 0.6 & $72 \cdot 9$ & 0.6 & $76 \cdot 6$ & 0.6 & 0.228 \\
\hline Model $2 \dagger$ & $72 \cdot 4$ & $1 \cdot 1$ & $72 \cdot 6$ & $1 \cdot 0$ & $73 \cdot 9$ & $1 \cdot 1$ & 0.534 \\
\hline \multicolumn{8}{|l|}{ Total fat $(\mathrm{g})$} \\
\hline Crude & $89 \cdot 5$ & $2 \cdot 2$ & 78.5 & $1 \cdot 6$ & $69 \cdot 6$ & $1 \cdot 7$ & $<0.001$ \\
\hline Model $1^{\star}$ & $81 \cdot 6$ & $0 \cdot 7$ & $79 \cdot 0$ & 0.7 & $78 \cdot 0$ & $0 \cdot 8$ & 0.001 \\
\hline Model $2 \dagger$ & 83.5 & 1.6 & 78.5 & 1.6 & $76 \cdot 2$ & 1.6 & 0.001 \\
\hline \multicolumn{8}{|l|}{ Fibre $(\mathrm{g})$} \\
\hline Crude & $22 \cdot 9$ & 0.5 & $23 \cdot 4$ & 0.5 & $24 \cdot 8$ & 0.5 & 0.001 \\
\hline Model $1^{\star}$ & $21 \cdot 2$ & 0.3 & $23 \cdot 5$ & 0.3 & $26 \cdot 6$ & 0.3 & $<0.001$ \\
\hline Model $2 \dagger$ & $21 \cdot 2$ & 0.4 & $23 \cdot 4$ & 0.4 & $26 \cdot 7$ & 0.4 & $<0.001$ \\
\hline \multicolumn{8}{|l|}{ Ethanol (g) } \\
\hline Crude & $14 \cdot 2$ & $1 \cdot 0$ & $10 \cdot 1$ & 0.6 & $8 \cdot 2$ & $0 \cdot 7$ & $<0.001$ \\
\hline Model $1^{\star}$ & $13 \cdot 1$ & 0.8 & $10 \cdot 1$ & 0.8 & $9 \cdot 3$ & $0 \cdot 8$ & $<0.001$ \\
\hline Model $2 \dagger$ & $14 \cdot 2$ & 0.8 & $10 \cdot 1$ & 0.8 & $8 \cdot 2$ & $0 \cdot 8$ & $<0.001$ \\
\hline
\end{tabular}

* Model 1: adjusted for energy intake estimated by the full-length FFQ.

† Model 2: adjusted for energy intake estimated by the DHD-FFQ.

wide limits of agreement between the DHD-index score derived from the DHD-FFQ and the reference method. Furthermore, the DHD-index score derived from the DHD-FFQ was positively associated with age, frequency of antihypertensive drug use and most micronutrient intakes, and was inversely associated with energy intake, whereas fasting TAG and HbA1C were non-significantly weakly associated.

We observed a Spearman's correlation of 0.57 (95\% CI 0.53 , 0.60) between the DHD-index score based on the DHD-FFQ data and the reference method, which was considered acceptable when assuming a maximum achievable correlation ranging between 0.66 and 0.72 . This maximum achievable strength of the correlation was based on the reproducibility of the DHD-index derived from a full-length FFQ after 1 year $(r 0.69 ; 95 \% \mathrm{CI} 0.64,0.74)$, the reproducibility of the 'Starting The Conversation' screener $(r 0.66)^{(5)}$ and the reproducibility of the 'Diet Quality Index Revised' $(r 0.72)^{(33)}$. The observed correlation in the present study was comparable with that of Schröder et $a l^{(6)}$ who observed a Pearson correlation coefficient of 0.52 comparing compliance with the PREDIMED dietary intervention derived from the fourteen-item 'Mediterranean Diet Adherence' screener and a 137-item FFQ. In another study by Schröder et al. ${ }^{(10)}$, a Pearson correlation coefficient of 0.61 was observed comparing the 'Diet Quality Index' derived from the 'Short Diet Quality' screener and derived from ten 24-h recalls. Furthermore, a correlation of 0.40 was observed for the 'Modified Mediterranean Diet Score' derived from the 'Brief Mediterranean Diet Score' screener compared with the score derived from ten 24-h recalls ${ }^{(10)}$.

Absolute agreement using the Bland-Altman plot showed a small mean overestimation of the DHD-index score derived from the DHD-FFQ compared with the DHD-index score derived from the reference method. Diet quality estimated by the DHD-FFQ was therefore considered acceptable on a group level. The Bland-Altman plot showed, furthermore, relatively wide limits of agreement. More extensive dietary assessment methods may therefore be needed when using individual scores for follow-up activities such as dietary advice. Taking together the results from ranking and absolute agreement, it suggests that the DHD-index score based on DHD-FFQ data can be used for ranking of participants and identification of high-risk subpopulations according to their diet quality.

For the components dietary fibre, SFA and TFA, the correlations between the DHD-index score based on the DHD-FFQ data and the reference method were lower than the expected value of $0 \cdot 4$. These low correlations could be explained by the lower percentages of MOM2 (47-65\%) covered by the DHD-FFQ for these components compared with MOM2 (100\%) for fruit, vegetables, fish and alcohol. The full-length FFQ showed considerably higher MOM2 percentages for the nutrients dietary fibre, SFA and TFA (>97\%), meaning that the DHD-FFQ estimates about $25 \%$ less variation of nutrient intakes compared with the full-length FFQ. Therefore, estimates for the component scores dietary fibre, SFA and TFA derived by the DHD-FFQ should be used carefully.

The component $\mathrm{Na}$ derived from the DHD-FFQ was compared with the score based on 24-h urinary $\mathrm{Na}$ value, and showed a low correlation of $0 \cdot 24$. It is known that estimating $\mathrm{Na}$ intake using a FFQ is challenging, which might explain the low correlation. However, our results might also be attenuated, because we included a single excretion and $\mathrm{Na}$ excretion is known to have a high within-person variability ${ }^{(34)}$. 
Table 5. Micronutrient intakes estimated from the full-length FFQ across quintiles (Q) of the Dutch Healthy Diet index (DHD-index) based on the DHD-FFQ in 1235 participants of the Nutrition Questionnaires plus study

(Mean values with their standard errors; $n$ 247)

\begin{tabular}{|c|c|c|c|c|c|c|c|}
\hline & \multicolumn{6}{|c|}{ DHD-index derived from the DHD-FFQ } & \multirow[b]{3}{*}{$P_{\text {for trend }}$} \\
\hline & \multicolumn{2}{|c|}{ Q1 } & \multicolumn{2}{|c|}{ Q3 } & \multicolumn{2}{|c|}{ Q5 } & \\
\hline & Mean & SEM & Mean & SEM & Mean & SEM & \\
\hline \multicolumn{8}{|l|}{$\mathrm{Ca}(\mathrm{mg})$} \\
\hline Crude & 979.0 & $25 \cdot 1$ & 933.9 & 20.5 & $885 \cdot 2$ & $19 \cdot 0$ & 0.001 \\
\hline Model $1^{\star}$ & $917 \cdot 0$ & $17 \cdot 2$ & 937.9 & $17 \cdot 0$ & 951.7 & $17 \cdot 2$ & 0.085 \\
\hline Model $2 \dagger$ & $916 \cdot 0$ & $19 \cdot 8$ & 934.4 & $19 \cdot 4$ & $956 \cdot 1$ & $19 \cdot 9$ & $0 \cdot 151$ \\
\hline \multicolumn{8}{|c|}{ Vitamin A (RAE) } \\
\hline Crude & $1038 \cdot 2$ & 44.9 & 930.6 & $32 \cdot 4$ & $900 \cdot 3$ & $30 \cdot 3$ & 0.001 \\
\hline Model $1^{*}$ & $964 \cdot 0$ & 34.5 & 935.4 & $34 \cdot 2$ & $980 \cdot 0$ & 34.5 & 0.886 \\
\hline Model $2 \dagger$ & 949.4 & $36 \cdot 1$ & 931.4 & $35 \cdot 3$ & $1000 \cdot 3$ & $36 \cdot 3$ & 0.692 \\
\hline \multicolumn{8}{|l|}{ Folate $(\mu \mathrm{g})$} \\
\hline Crude & $254 \cdot 2$ & 5.8 & 265.5 & $6 \cdot 1$ & $291 \cdot 2$ & $6 \cdot 0$ & $<0.001$ \\
\hline Model $1^{*}$ & $237 \cdot 6$ & 4.7 & $266 \cdot 6$ & 4.6 & $309 \cdot 1$ & 4.7 & $<0.001$ \\
\hline Model 2† & $237 \cdot 2$ & $5 \cdot 4$ & $265 \cdot 7$ & $5 \cdot 3$ & $310 \cdot 4$ & 5.4 & $<0.001$ \\
\hline \multicolumn{8}{|c|}{ Vitamin $B_{1}(m g)$} \\
\hline Crude & 1.03 & 0.02 & 1.00 & 0.02 & 0.93 & 0.02 & $<0.001$ \\
\hline Model $1^{*}$ & 0.96 & 0.01 & 1.00 & 0.01 & 1.01 & 0.01 & 0.001 \\
\hline Model 2† & 0.96 & 0.02 & 1.00 & 0.02 & 1.01 & 0.02 & 0.055 \\
\hline \multicolumn{8}{|c|}{ Vitamin $B_{2}(m g)$} \\
\hline Crude & 1.48 & 0.03 & 1.44 & 0.03 & 1.37 & 0.03 & 0.001 \\
\hline Model $1^{*}$ & 1.38 & 0.02 & 1.45 & 0.02 & 1.48 & 0.02 & 0.007 \\
\hline Model $2 \dagger$ & 1.38 & 0.03 & 1.44 & 0.03 & 1.49 & 0.03 & 0.028 \\
\hline \multicolumn{8}{|c|}{ Vitamin $B_{6}(\mathrm{mg})$} \\
\hline Crude & 1.60 & 0.03 & 1.55 & 0.03 & 1.55 & 0.03 & 0.247 \\
\hline Model $1^{*}$ & 1.48 & 0.02 & 1.56 & 0.02 & 1.68 & 0.02 & $<0.001$ \\
\hline Model 2† & 1.49 & 0.03 & 1.55 & 0.03 & 1.67 & 0.03 & $<0.001$ \\
\hline \multicolumn{8}{|c|}{ Vitamin $B_{12}(\mu \mathrm{g})$} \\
\hline Crude & $4 \cdot 2$ & 0.1 & 4.0 & 0.1 & 4.0 & 0.1 & 0.096 \\
\hline Model $1^{*}$ & $4 \cdot 0$ & $0 \cdot 1$ & $4 \cdot 0$ & 0.1 & $4 \cdot 3$ & 0.1 & 0.046 \\
\hline Model $2 \dagger$ & $4 \cdot 0$ & 0.1 & 4.0 & 0.1 & 4.4 & 0.1 & 0.062 \\
\hline \multicolumn{8}{|c|}{ Vitamin C (mg) } \\
\hline Crude & 57.5 & $2 \cdot 1$ & 87.5 & $2 \cdot 4$ & 98.5 & 2.5 & $<0.001$ \\
\hline Model $1^{\star}$ & $64 \cdot 1$ & $2 \cdot 1$ & $87 \cdot 7$ & $2 \cdot 1$ & $103 \cdot 2$ & $2 \cdot 1$ & $<0.001$ \\
\hline Model 2† & $64 \cdot 3$ & $2 \cdot 3$ & 87.5 & $2 \cdot 2$ & $103 \cdot 3$ & $2 \cdot 3$ & $<0.001$ \\
\hline \multicolumn{8}{|c|}{ Vitamin $E(m g)$} \\
\hline Crude & $13 \cdot 0$ & 0.3 & $12 \cdot 7$ & 0.3 & 12.5 & 0.3 & 0.210 \\
\hline Model $1^{*}$ & 11.9 & 0.2 & $12 \cdot 8$ & 0.2 & $13 \cdot 8$ & 0.2 & $<0.001$ \\
\hline Model $2 \dagger$ & $12 \cdot 0$ & 0.3 & $12 \cdot 7$ & 0.3 & $13 \cdot 7$ & 0.3 & $<0.001$ \\
\hline
\end{tabular}

RAE, retinol active equivalents $(\mu \mathrm{g})$.

* Model 1: adjusted for energy intake estimated by the full-length FFQ.

† Model 2: adjusted for energy intake estimated by the DHD-FFQ.

When comparing the $\mathrm{Na}$ component based on the full-length FFQ data with the $\mathrm{Na}$ component based on 24-h urinary excretion data, the correlation was as low as that for the DHD-FFQ data $(0 \cdot 20 v \cdot 0 \cdot 24)$. Furthermore, when comparing the $\mathrm{Na}$ component derived from the full-length FFQ with the $\mathrm{Na}$ component derived from the DHD-FFQ, the correlation was acceptable $(0.64 ; 95 \%$ CI $0.61,0.68)$. These results suggest that the DHD-FFQ estimates $\mathrm{Na}$ intake with accuracy similar to that of the full-length FFQ and both showed only a moderate association with a single urinary $\mathrm{Na}$ excretion value. Conclusions on the $\mathrm{Na}$ component score, estimated either by DHD-FFQ or full-length FFQ, should therefore be drawn with caution.

The DHD-index derived from the DHD-FFQ was associated with sex, age, following a diet regimen, supplement use, smoking and antihypertensive medication use. The DHD-index scores based on a full-length FFQ and on two 24-h recalls showed similar associations with sex ${ }^{(3,4,35)}$, age ${ }^{(4,35)}$, smoking ${ }^{(35)}$ and following a diet regimen ${ }^{(3)}$ in other Dutch populations. However, in these other populations, the DHD-index was also associated with higher education ${ }^{(35)}$, which was not seen in the present study. This discrepancy may be explained by the high percentage of highly educated participants (53.9\%) in the present study.

Intakes of most macronutrients and micronutrients showed associations with the DHD-index derived from the DHD-FFQ in the expected direction when we adjusted for energy intake estimated by either the full-length FFQ or the DHD-FFQ. Although the DHD-FFQ was not designed to estimate energy intake and the estimated energy intake coverage was low (64\%), the estimated energy intake was moderate-to-highly correlated with energy intake estimated by the full-length FFQ ( $r$ 0.60; 95\% CI 0.57, 0.64). Others found correlations ranging between 0.46 and 0.58 when comparing several FFQ with 24-h recalls or food records ${ }^{(36)}$. The present correlation shows a good ranking capacity, and therefore the energy intake estimate may be used as a covariate, making the DHD-FFQ useful for 
epidemiological research. The favourable associations of the DHD-index with nutrient intakes were also observed in the DNFCS 2003 using two 24-h recalls to calculate the DHD-index score $^{(3)}$ and in the European Food Consumption Validation study using a 180-item FFQ ${ }^{(4)}$. We also showed that the DHD-index derived from the DHD-FFQ was able to reflect associations with nutrient intakes in a favourable direction.

The DHD-index derived from both the DHD-FFQ and the reference method showed weak inverse associations with fasting TAG and HbA1C. The weak and absent associations between diet quality and cardiometabolic risk factors could be partly due to the relative good health of our participants. Among participants with high risk for CHD, Schröder et al. ${ }^{(6)}$ observed significant associations of HDL-cholesterol $(\beta=0 \cdot 01)$, TAG $(\beta=-0.005)$ and fasting glucose $(\beta=-0.003)$ with adherence to the Mediterranean diet using a fourteen-item questionnaire. Associations between diet quality as assessed by a full-length FFQ or multiple recalls with cardiometabolic risk factors ranged between 0.02 and 0.13 and $\beta$ s ranged between 0.00 and 0.44 across studies ${ }^{(37)}$.

We aimed to keep the calculation of the DHD-index components based on the DHD-FFQ as similar to the original calculation as possible; however, we had to make some adjustments. First, instead of lowering the cut-off values for the $\mathrm{Na}$ component by $30 \%$, as was done previously ${ }^{(3,4)}$, we aimed to estimate discretionary $\mathrm{Na}$ by adding two questions on salt use during cooking and the use of salt or Na-rich products at the table that accounted for 3 points out of the maximum of 10 points. When excluding these questions from the $\mathrm{Na}$ component calculation, the correlation with the urinary $\mathrm{Na}$ component was similar $(0.24 v \cdot 0 \cdot 25)$. These findings were supported by other studies, showing that questions on salt preference and discretionary salt poorly estimated $\mathrm{Na}$ intake ${ }^{(38,39)}$. The reason why the additional questions on discretionary $\mathrm{Na}$ did not improve the correlation with the urinary $\mathrm{Na}$ component is unclear and needs further investigation. Second, the energy-dependent cut-off values for the components fibre, SFA and TFA were based on average energy requirements used in the Netherlands, because the DHD-FFQ was not designed to estimate energy intake. Furthermore, the cutoff values for the components fibre, SFA and TFA were lowered in accordance with the estimated percentage of coverage for the energy intake (64\%) assessed by the DHD-FFQ. Lowering the cut-off values was chosen because otherwise participants could not receive the maximum or minimum number of points as the DHD-FFQ did not assess the complete dietary intake. However, due to the likely individual deviations from the average energy intake requirements, misclassifications could have been introduced to the DHD-index scores.

A limitation of the present study may be the large proportion of the study population who were educated to a high level, meaning that the results of the usability of the DHD-FFQ may not be generalisable to populations with a lower educational level. Furthermore, all the participants were also involved in completing multiple 24-h recalls, which might have led to a training effect. Moreover, the web-based administration of the full-length FFQ and the DHD-FFQ was not validated, and disadvantages such as lower reliability and validity of data obtained have been suggested ${ }^{(40)}$. However, Beasley et al. ${ }^{(41)}$ showed that web-based administration of FFQ produce similar results as paper-based administration. Furthermore, advantages such as restriction for the range of answer possibilities and obligatory questions were also mentioned ${ }^{(40)}$. The possible disadvantages and advantages might have affected both FFQ similarly, and thus it is unlikely that it affected our results.

Another limitation was the order of occurrence of the DHDFFQ and the full-length FFQ, which was not evenly distributed. However, additional adjustments for order of occurrence and time between the two methods did not alter our correlation coefficients notably.

One of the strengths of the present study was the large study population used to evaluate the DHD-index derived from the DHD-FFQ. Furthermore, the reference method consisted of a 180-item FFQ, validated for energy, macronutrients and micronutrients ${ }^{(11,13)}$

In conclusion, the DHD-index score based on the DHD-FFQ was considered an acceptable screener to rank participants according to their diet quality and was associated with several participant characteristics and macronutrient and micronutrient intakes in the present study. The assessment of individual diet quality scores by the DHD-FFQ was relatively poor as was shown by the large limits of agreement.

\section{Acknowledgements}

The work was supported by ZonMw (grant number 115100007), The Netherlands Organization for Health Research and Development, The Hague. The NQplus study was funded by ZonMw (grant number 91110030) and Wageningen University. The sponsors had no role in the study design, data collection, analysis and interpretation of the data, and writing of the report.

L. v. L. participated in the manuscript conception, statistical analyses, data interpretation, manuscript writing and revising; E. J. M. F. and A. G. participated in the manuscript conception, data interpretation, manuscript writing and review; S. M., E. J. C. H. v. H., P. v. V. and J. H. M. d. V. contributed to data interpretation and review. All the authors read and approved the final version of the manuscript.

The authors declare that there are no conflicts of interest.

\section{Supplementary material}

For supplementary material/s referred to in this article, please visit http://dx.doi.org/doi:10.1017/S0007114515004705

\section{References}

1. WHO Regional Office for Europe (2008) WHO European Action Plan for Food and Nutrition Policy 2007-2012. Copenhagen: WHO.

2. Health Council of the Netherlands (2006) Guidelines for a Healthy Diet 2006. Publication no. 2006/21. The Hague: Health Council of the Netherlands.

3. van Lee L, Geelen A, van Huysduynen EJ, et al. (2012) The Dutch Healthy Diet index (DHD-index): an instrument to measure adherence to the Dutch guidelines for a healthy diet. Nutr J 11, 1-9. 
4. van Lee L, Feskens EJ, Hooft van Huysduynen EJ, et al. (2014) The Dutch Healthy Diet index as assessed by $24 \mathrm{~h}$ recalls and FFQ: associations with biomarkers from a cross-sectional study. J Nutr Sci 2, e40.

5. Paxton AE, Strycker LA, Toobert DJ, et al. (2011) Starting the conversation performance of a brief dietary assessment and intervention tool for health professionals. Am J Prev Med 40, 67-71.

6. Schröder H, Fitó M, Estruch R, et al. (2011) A short screener is valid for assessing Mediterranean diet adherence among older Spanish men and women. $J$ Nutr 141, 1140-1145.

7. Mochari H, Gao Q \& Mosca L (2008) Validation of the MEDFICTS dietary assessment questionnaire in a diverse population. J Am Diet Assoc 108, 817-822.

8. Rifas-Shiman SL, Willett WC, Lobb R, et al. (2001) PrimeScreen, a brief dietary screening tool: reproducibility and comparability with both a longer food frequency questionnaire and biomarkers. Public Health Nutr 4, 249-254.

9. Apovian CM, Murphy MC, Cullum-Dugan D, et al. (2010) Validation of a web-based dietary questionnaire designed for the DASH (Dietary Approaches to Stop Hypertension) diet: the DASH online questionnaire. Public Health Nutr 13, 615-622.

10. Schröder H, Benitez Arciniega A, Soler C, et al. (2012) Validity of two short screeners for diet quality in time-limited settings. Public Health Nutr 15, 618-626.

11. Siebelink E, Geelen A \& de Vries JHM (2011) Self-reported energy intake by FFQ compared with actual energy intake to maintain body weight in 516 adults. Br J Nutr 106, 274-281.

12. Feunekes GI, Van Staveren WA, De Vries JH, et al. (1993) Relative and biomarker-based validity of a food-frequency questionnaire estimating intake of fats and cholesterol. $\mathrm{Am}$ J Clin Nutr 58, 489-496.

13. Streppel MT, De Vries JH, Meijboom S, et al. (2013) Relative validity of the food frequency questionnaire used to assess dietary intake in the Leiden Longevity Study. Nutr J 12, 1-8.

14. Wendel-Vos GCW, Schuit AJ, Saris WHM, et al. (2003) Reproducibility and relative validity of the short questionnaire to assess health-enhancing physical activity. J Clin Epidemiol 56, 1163-1169.

15. Molag ML (2010) Towards transparent development of food frequency questionnaires. Scientific basis of the Dutch FFQ-TOOL $^{\mathrm{TM}}$ : a computer system to generate, apply and process FFQs. Thesis, Wageningen University, Wageningen.

16. van Rossum CTM, Fransen HP, Verkaik-Kloosterman J, et al. (2011) Dutch National Food Consumption Survey 2007-2010, RIVM Report 350050006/2011. Bilthoven, the Netherlands: National Institute for Public Health and the Environment.

17. Block G, Dresser CM, Hartman AM, et al. (1985) Nutrient sources in the American diet: quantitative data from the NHANES II survey. I. Vitamins and minerals. Am J Epidemiol 122, 27-40.

18. Block G, Hartman AM, Dresser CM, et al. (1986) A data-based approach to diet questionnaire design and testing. Am J Epidemiol 124, 453-469.

19. Mark SD, Thomas DG \& Decarli A (1996) Measurement of exposure to nutrients: an approach to the selection of informative foods. Am J Epidemiol 143, 514-521.

20. NEVO-tabel (2011) Dutch Food Composition Table 2011/ version 3. Bilthoven, the Netherlands: RIVM/Dutch Nutrition Centre.

21. Anonymous (1998) Basisrapportage en tabellen voedselconsumptiepeiling 1997-1998 en gegevens op het niveau van voedingsmiddelen en voedingsstoffen over een periode van tien jaar (Report and Tables of the Food Consumption Survey 1997-1998 and Information on Foods and Nutrients Over a Period of 10 Years). Zeist: TNO Voeding.
22. Brown IJ, Tzoulaki I, Candeias V, et al. (2009) Salt intakes around the world: implications for public health. Int J Epidemiol 38, 791-813.

23. Jakobsen J, Ovesen L, Fagt S, et al. (1997) Para-aminobenzoic acid used as a marker for completeness of 24 hour urine: assessment of control limits for a specific HPLC method. Eur J Clin Nutr 51, 514-519.

24. Johansson G, Bingham S \& Vahter M (1999) A method to compensate for incomplete 24-hour urine collections in nutritional epidemiology studies. Public Health Nutr 2, 587-591.

25. Hagfors L, Westerterp K, Skoldstam L, et al. (2005) Validity of reported energy expenditure and reported intake of energy, protein, sodium and potassium in rheumatoid arthritis patients in a dietary intervention study. Eur J Clin Nutr 59, 238-245.

26. Holbrook JT, Patterson KY \& Bodner JE (1984) Sodium and potassium intake and balance in adults consuming selfselected diets. Am J Clin Nutr 40, 786-793.

27. Mulder M (2013) Zware drinkers 2012 (Heavy drinkers 2012). In Volksgezondheid Toekomst Verkenning, Nationale Atlas Volksgezondheid.

Bilthoven: RIVM.

28. Health Council of the Netherlands (2001) Dietary Reference Intakes: Energy, Proteins, Fats and Digestible Carbohydrates. Publication no. 2001/19R. The Hague: Health Council of the Netherlands.

29. Mattes RD \& Donnelly D (1991) Relative contributions of dietary sodium sources. J Am Coll Nutr 10, 383-393.

30. Health Council of the Netherlands (2000) Salt and Blood Pressure. Publication no. 2000/13. The Hague: Health Council of the Netherlands.

31. Allain CC, Poon LS, Chan CSG, et al. (1974) Enzymatic determination of total serum cholesterol. Clin Chem 20, 470-475.

32. Bland JM \& Altman DG (1986) Statistical methods for assessing agreement between two methods of clinical measurement. Lancet i, 307-310.

33. Newby PK, Hu FB, Rimm EB, et al. (2003) Reproducibility and validity of the diet quality index revised as assessed by use of a food-frequency questionnaire. Am J Clin Nutr 78, 941-949.

34. Bingham SA, Williams R, Cole TJ, et al. (1988) Reference values for analytes of 24-h urine collections known to be complete. Ann Clin Biochem 25, 610-619.

35. Struijk EA, May AM, Beulens JW, et al. (2014) Adherence to the Dutch guidelines for a healthy diet and cancer risk in the European Prospective Investigation into Cancer and Nutrition-Netherlands (EPIC-NL) cohort. Public Health Nutr 17, 2546-2553.

36. Molag ML, De Vries JHM, Ocké MC, et al. (2007) Design characteristics of food frequency questionnaires in relation to their validity. Am J Epidemiol 166, 1468-1478.

37. Bondia-Pons I, Mayneris-Perxachs J, Serra-Majem L, et al. (2010) Diet quality of a population sample from coastal northeast Spain evaluated by a Mediterranean adaptation of the diet quality index (DQI). Public Health Nutr 13, 12-24.

38. Hashimoto T, Yagami F, Owada M, et al. (2008) Salt preference according to a questionnaire vs. dietary salt intake estimated by a spot urine method in participants at a health check-up center. Intern Med 47, 399-403.

39. Charlton KE, Steyn K, Levitt NS, et al. (2008) Development and validation of a short questionnaire to assess sodium intake. Public Health Nutr 11, 83-94.

40. van Gelder MM, Bretveld RW \& Roeleveld N (2010) Web-based questionnaires: the future in epidemiology? $\mathrm{Am}$ J Epidemiol 172, 1292-1298.

41. Beasley JM, Davis A \& Riley WT (2009) Evaluation of a webbased, pictorial diet history questionnaire. Public Health Nutr 12, 651-659. 